Review Article

\title{
Single- versus Multiple-Tract Percutaneous Nephrolithotomy in the Surgical Management of Staghorn Stones or Complex Caliceal Calculi: A Systematic Review and Meta-analysis
}

\author{
Binbin Jiao, ${ }^{1,2}$ Zhenshan Ding, ${ }^{2}$ Zhenkai Luo, ${ }^{2,3}$ Shicong Lai, ${ }^{1,4} \mathrm{Xin} \mathrm{Xu}^{2}{ }^{2} \mathrm{Xing}$ Chen, ${ }^{2}$ \\ and Guan Zhang $\mathbb{1}^{1,2,3}$ \\ ${ }^{1}$ Graduate School of Peking Union Medical College and Chinese Academy of Medical Sciences, Beijing, China 100029 \\ ${ }^{2}$ Department of Urology, China-Japan Friendship Hospital, Yinghuadong Road, Chaoyang District, Beijing 100029, China \\ ${ }^{3}$ Peking University China-Japan Friendship School of Clinical Medicine, Yinghuadong Road, Chaoyang District, \\ Beijing 100029, China \\ ${ }^{4}$ Department of Urology, Beijing Hospital, No. 1, Dahua Road, Dongdan, Dongcheng District, Beijing 100005, China
}

Correspondence should be addressed to Guan Zhang; gzhang2016@sina.com

Received 6 September 2020; Revised 18 November 2020; Accepted 30 November 2020; Published 18 December 2020

Academic Editor: Riccardo Campi

Copyright (c) 2020 Binbin Jiao et al. This is an open access article distributed under the Creative Commons Attribution License, which permits unrestricted use, distribution, and reproduction in any medium, provided the original work is properly cited.

Objective. To assess current evidence on the effectiveness and safety of single- versus multiple-tract percutaneous nephrolithotomy in the surgical management of complex caliceal calculi or staghorn stones through a comprehensive literature review. Methods. A comprehensive literature review of articles investigating the clinical efficacy and safety of single- versus multiple-tract percutaneous nephrolithotomy was performed. Relevant literature was obtained by systematically searching PubMed, EMBASE, and the Cochrane Library through May 2020. We followed the search strategy based on the Preferred Reporting Items for Systematic Reviews and Meta-Analyses statement. The primary outcomes, including the stone-free rate (SFR), and secondary outcomes (peri- and postoperative complications and operative data) were evaluated using RevMan 5.3 statistical software. Results. Ten studies involving 1844 patients with complex caliceal calculi or staghorn stones met the inclusion criteria. Single-tract percutaneous nephrolithotomy (STPCNL) had noninferior clinical efficacy with respect to the immediate SFR (odds ratio $(\mathrm{OR})=0.80,95 \%$ confidence interval $(\mathrm{CI})(0.46$ to 1.38$), p=0.42)$ and 3 -month SFR $(\mathrm{OR}=1.22,95 \% \mathrm{CI}(0.38$ to 3.92), $p=0.74)$ compared with multiple-tract percutaneous nephrolithotomy (MTPCNL). In addition, pooled analyses showed that STPCNL resulted in significantly lower hemoglobin decreases $(\mathrm{MD}=-0.46,95 \% \mathrm{CI}(-0.68$ to -0.25$), p<0.0001)$, fewer blood transfusions ( $\mathrm{OR}=0.48,95 \% \mathrm{CI}(0.34$ to 0.67$), p<0.0001)$, and fewer pulmonary complications $(\mathrm{OR}=0.28,95 \% \mathrm{CI}(0.09$ to 0.83$), p=0.02$ ) than MTPCNL. However, the overall evidence was insufficient to suggest a statistically significant difference for other adverse events. Conclusion. This meta-analysis indicated that STPCNL is an effective method for treating complex caliceal calculi or staghorn stones. Compared with MTPCNL, STPCNL not only yields similarly high SFRs but also is associated with many advantages, less blood loss, fewer blood transfusions, and fewer pulmonary complications without an increase in other complications. However, the findings of this study should be further confirmed by well-designed prospective randomized controlled trials (RCTs) with a larger patient series.

\section{Introduction}

Ureteral calculi represent a common disease that seriously endangers life and work for more than $12 \%$ of the population. Staghorn or complex caliceal calculi constitute one of the most challenging problems in urology and are likely to destroy the function of the kidney and cause lifethreatening sepsis [1]. For patients with staghorn or complex caliceal calculi, the goal of treatment is to achieve maximal clearance of stones and assure maximal renal function preservation with minimal complications. In the recently updated guidelines of the American Urological Association 
Nephrolithiasis Guideline Panel on Staghorn Calculi, percutaneous nephrolithotomy (PCNL) is an integral component of the management of most staghorn and large-volume renal calculi [2]. However, complex caliceal and staghorn stones are difficult to remove with a single-tract PCNL approach [3]. A trend toward the use of percutaneous monotherapy using multiple tracts as the preferred treatment option for most staghorn or complex calculi has emerged [4]. However, a concern with creating multiple percutaneous tracts is the potential risks of greater bleeding and higher complication rates compared with the single-tract approach [5]. Therefore, many urologists hesitate to place multiple percutaneous tracts during PCNL. In recent years, an increasing number of studies have been conducted to assess the clinical efficacy, operative results, and complications of STPCNL and MTPCNL, but the outcomes of these studies have varied. Thus, conducting a new systematic review and metaanalysis that includes relevant available studies evaluating the efficacy of single- versus multiple-tract PCNL in the surgical management of complex caliceal calculi or staghorn stones is worthwhile.

\section{Method}

2.1. Search Strategy. To assess the clinical efficacy and safety of single- versus multiple-tract PCNL, a comprehensive literature search was performed using PubMed, EMBASE, and the Cochrane Library in February 2020. We considered the definition of staghorn calculi to be stones that branched and occupied a large portion of the collecting system, such as complete staghorn stones (occupying the renal pelvis and the entire caliceal system or occupying $80 \%$ of the renal collecting system) or partial staghorn stones (occupying the renal pelvis or at least two or more calices), and we defined complex caliceal calculi as those with a large bulk and involving more than one calix, the upper ureter, or both. The keywords "single tract", "multiple tract", "percutaneous nephrolithotomy", "complex caliceal calculi", and "staghorn stones" were used to search for articles. These search terms were used individually and in combination. Additionally, manual searches of the references and citation lists of all relevant reviews were performed. For publication selection, a search strategy was applied based on the Preferred Reporting Items for Systematic Reviews and Meta-Analyses (PRISMA) statement and the assessing the methodological quality of systematic reviews (AMSTAR) guidelines.

Studies meeting the following criteria were included: (1) studies comparing the safety and efficacy of single- versus multiple-tract PCNL for surgical treatment of complex caliceal calculi or staghorn stones; (2) outcome measures consisting of at least one of the following treatment-related adverse events and functional outcomes: the stone-free rate (SFR), complications, hospitalization times, operative times, blood loss, and blood transfusion when available; and (3) articles written in English with the full text or related data available. The exclusion criteria were as follows: (1) duplicate publications or conference proceedings; (2) nonpublished materials, editorials, or reviews; and (3) studies containing patients with serious urinary infection, renal insufficiency, musculoskeletal deformities, solitary kidney, or congenital abnormalities.

Relevant references cited in the selected papers were also retrieved. The literature search and selection were independently performed by 3 reviewers (J.B., D.Z., and Z.L.) and then cross-checked. Any differences at this stage were resolved through discussion and by a majority decision of the reviewers if necessary. A flowchart showing the number of publications selected or excluded at each stage is presented in Figure 1. Ethics committee approval for this study was not necessary because all the data were carefully extracted from the existing literature, and this article did not involve individual patient data.

2.2. Assessment of Study Quality. We evaluated the level of evidence for each selected article based on the criteria recommended by the Oxford Centre for Evidence-based Medicine [6]. For the methodological quality assessment, we used the Newcastle-Ottawa Scale (NOS) [7] to evaluate the quality of prospective studies. In addition, we evaluated the methodological quality of the trials according to the methods recommended by the Cochrane Collaboration.

Preoperative parameters were extracted together with intraoperative data, including operation times, hemoglobin decreases, and transfusion rates. Postoperative data, including the SFR, length of hospitalization, and treatment-related complications, were also analyzed. Functional results, including renal function, were assessed after surgery.

Ten relevant studies [8-17] including 1844 patients were selected for analysis. No differences were found in terms of age and basic physical conditions between the MTPCNL and STPCNL groups. Data extraction was independently performed by 2 reviewers (J.B. and Z.L.) and then crosschecked. Any differences at this stage were resolved through discussion and by a majority decision of the reviewers if necessary.

We used the mean difference (MD) to evaluate continuous outcomes. For the studies expressing continuous data as the median and range values, we used the statistical formula described by Wan et al. [18] to determine the mean and standard deviation in accordance with the recommended methods described in the Cochrane Handbook for Systematic Reviews 23.

The results are expressed as the odds ratio (OR) with a 95\% confidence interval (CI) for dichotomous variables. The $\chi^{2}$ and $I^{2}$ tests were used to assess the heterogeneity of the study data $\left(I^{2}>50 \%\right.$ was regarded as substantial heterogeneity). If the heterogeneity was considered low, fixed effects models were used for the meta-analyses. Otherwise, a random effects model was used to reduce the effect of statistical heterogeneity. The pooled effects were determined by a $z$ test, and a $p$ value $<0.05$ was considered statistically significant. Moreover, for the comparisons of MTPCNL and STPCNL, relevant publications with appropriate data allowed us to perform subgroup analyses according to the device used. For several comparisons, sensitivity analyses were used. The meta-analysis of comparable data was performed using Review Manager (RevMan) 5.3 software. 


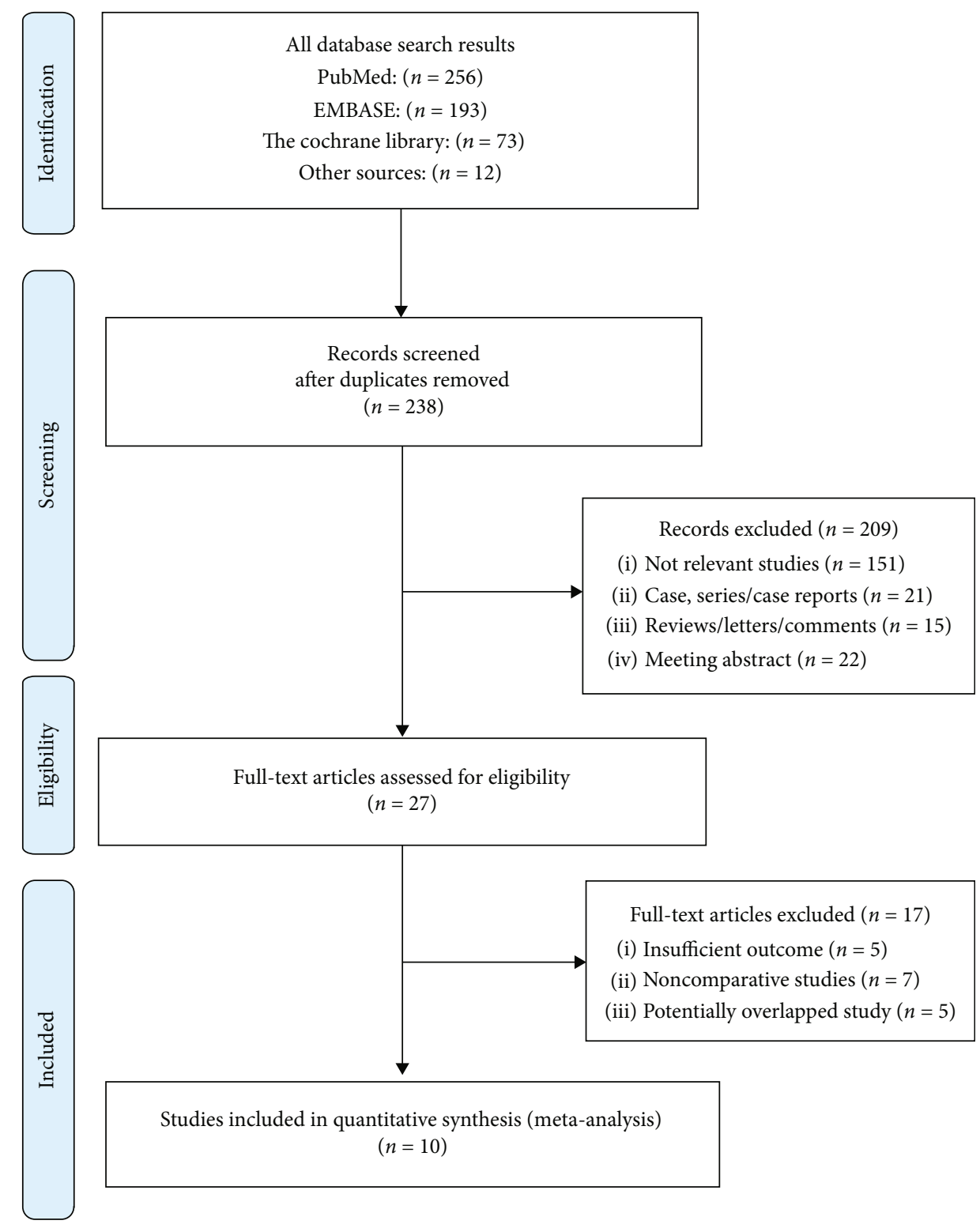

FIgURE 1: Study selection flowchart.

\section{Results}

The initial search strategy yielded 534 studies from the metadatabases combined. Our strict eligibility criteria resulted in the exclusion of 524 reports. Ten studies focusing on two different complex caliceal calculus or staghorn stone interventions were included, which involved 1844 participants, $48.26 \% \quad(n=890)$ of whom underwent STPCNL, while $51.73 \%(n=954)$ underwent MTPCNL. Examination of the references listed for these studies and for the review articles did not yield any further studies for evaluation.

3.1. Characteristics of the Selected Studies. Patient characteristics and study characteristics are summarized in Table 1 . No differences were found in basic physical conditions for all of the included studies. The outcome parameters for the differ- ential management of complex caliceal calculi or staghorn stones are shown in Table 2.

3.2. SFR. Three included studies compared the immediate SFRs of single- versus multiple-tract PCNL. The random effects model was selected for analysis due to significant heterogeneity among these trials $\left(I^{2}=59 \%\right)$. The overall results showed no significant difference between single- and multiple-tract PCNL for the immediate SFR $(\mathrm{OR}=0.80$, $95 \%$ CI (0.46 to 1.38$), p=0.42$ ) (Figure 2(a)). In addition, a random effects model was used to analyze the 3-month SFR. No significant difference was found between the singleand multiple-tract PCNL SFRs (OR $=1.22,95 \%$ CI ( 0.38 to 3.92), $p=0.74$ ) (Figure 2(a)). The sensitivity analysis suggested the same results. 
TABLE 1: Summary of comparative studies included in meta-analysis.

\begin{tabular}{|c|c|c|c|c|c|c|c|c|c|}
\hline \multirow{2}{*}{ Study } & \multirow{2}{*}{ Country } & \multirow{2}{*}{ Study period } & \multirow{2}{*}{ Study design } & \multirow{2}{*}{ LE } & \multicolumn{2}{|c|}{ Intervention } & \multicolumn{2}{|c|}{ Sample size } & \multirow{2}{*}{ Study quality } \\
\hline & & & & & Trial & Control & Trial & Control & \\
\hline Blum 2018 & America & Not mentioned & Prospective study & $2 \mathrm{~b}$ & STPCNL & MTPCNL & 57 & 19 & $8^{\#}$ \\
\hline Nahas 2012 & Egypt & $1999-2009$ & Not mentioned & $2 \mathrm{~b}$ & STPCNL & MTPCNL & 156 & 86 & $7^{\#}$ \\
\hline Mishra 2012 & India & 2009-2010 & Retrospective study & $2 \mathrm{a}$ & STPCNL & MTPCNL & 41 & 53 & $7^{\#}$ \\
\hline Akman 2010 & Turkey & 2002-2009 & Retrospective study & $2 \mathrm{a}$ & STPCNL & MTPCNL & 244 & 169 & $7^{\#}$ \\
\hline Hegarty 2006 & America & 2004-2005 & Retrospective study & $2 \mathrm{a}$ & STPCNL & MTPCNL & 20 & 20 & $7^{\#}$ \\
\hline Desai 2004 & India & 1991-2002 & Retrospective study & $2 b$ & STPCNL & MTPCNL & 22 & 34 & $6^{\#}$ \\
\hline Cho 2012 & Korea & 2003-2008 & Not mentioned & $2 b$ & STPCNL & MTPCNL & 79 & 30 & $7^{\#}$ \\
\hline Ozden 2008 & Turkey & $2000-2005$ & Retrospective study & $2 b$ & STPCNL & MTPCNL & 32 & 21 & $7^{\#}$ \\
\hline Desai 2008 & India & $1991-2007$ & Retrospective study & $2 b$ & STPCNL & MTPCNL & 225 & 500 & $8^{\#}$ \\
\hline Manohar 2006 & India & $1991-2004$ & Not mentioned & $2 \mathrm{a}$ & STPCNL & MTPCNL & 14 & 22 & $7^{\#}$ \\
\hline
\end{tabular}

$\mathrm{LE}=$ level of evidence; STPCNL = single-tract percutaneous nephrolithotomy; MTPCNL = multiple-tract percutaneous nephrolithotomy. ${ }^{*}$ Using NewcastleOttawa Scale (score from 0 to 9 ).

TABLE 2: Study outcomes comparing STPCNL and MTPCNL.

\begin{tabular}{|c|c|c|c|c|c|c|c|c|c|}
\hline \multirow{2}{*}{ Outcomes } & \multirow{2}{*}{ No. of studies } & \multicolumn{2}{|c|}{ Sample size } & \multicolumn{4}{|c|}{ Heterogeneity (total) } & \multirow{2}{*}{$\mathrm{MD}$ or $\mathrm{OR}(95 \% \mathrm{CI})$} & \multirow{2}{*}{$p$ value (total) } \\
\hline & & STPCNL & MTPCNL & $\mathrm{Chi}^{2}$ & $\mathrm{df}$ & $I^{2}(\%)$ & $p$ value & & \\
\hline Initial SFR & 3 & 543 & 699 & 4.84 & 2 & 59 & 0.09 & $0.80(0.46,1.38)$ & $p=0.42$ \\
\hline 3-month SFR & 2 & 364 & 252 & 5.10 & 2 & 61 & 0.08 & $1.22(0.38,3.92)$ & $p=0.74$ \\
\hline Auxiliary treatment & 3 & 343 & 219 & 38.51 & 2 & 95 & $<0.00001$ & $0.34(0.03,3.96)$ & $p=0.39$ \\
\hline Hemoglobin drop & 4 & 493 & 307 & 0.68 & 3 & 0 & 0.88 & $-0.47(-0.68,-0.25)$ & $p<0.0001$ \\
\hline Blood transfusion & 5 & 657 & 759 & 3.6 & 4 & 0 & 0.46 & $0.48(0.34,0.67)$ & $p<0.0001$ \\
\hline Operation time & 2 & 323 & 199 & 7.74 & 1 & 87 & 0.005 & $-42.78(-85.49,0.07)$ & $p=0.05$ \\
\hline Hospital stay & 3 & 140 & 103 & 15.23 & 2 & 87 & 0.0005 & $-0.59(-3.59,2.41)$ & $p=0.70$ \\
\hline Postoperative fever & 2 & 136 & 49 & 0.01 & 1 & 0 & 0.9 & $0.86(0.27,2.78)$ & $p=0.8$ \\
\hline Renal function & 3 & 323 & 199 & 0.23 & 1 & 0 & 0.63 & $-0.02(-0.06,0.02)$ & $p=0.32$ \\
\hline Urinary leakage & 4 & 402 & 252 & 1.45 & 3 & 0 & 0.69 & $0.60(0.19,1.87)$ & $p=0.38$ \\
\hline Urinary tract infection & 3 & 99 & 73 & 0.02 & 1 & 0 & 0.89 & $0.85(0.13,5.45)$ & $p=0.87$ \\
\hline Complications & 4 & 480 & 636 & 0.97 & 3 & 0 & 0.81 & $0.74(0.49,1.12)$ & $p=0.15$ \\
\hline Pulmonary complications & 3 & 321 & 208 & 1.71 & 2 & 0 & 0.42 & $0.28(0.09,0.83)$ & $p=0.02$ \\
\hline Nephrostomy time & 2 & 323 & 199 & 3.23 & 1 & 69 & 0.07 & $0.94(-2.39,4.26)$ & $p=0.58$ \\
\hline
\end{tabular}

$\mathrm{CI}=$ confidence interval; $\mathrm{MD}=$ mean difference; $\mathrm{RR}=$ risk ratio.

3.3. Auxiliary Treatment. Auxiliary procedures to achieve stone-free status included shockwave lithotripsy, "sandwich therapy," repeat PCNL, and URS after STPCNL or MTPCNL. The use of such procedures was reported in two studies focusing on STPCNL and MTPCNL. A pooled analysis showed no significant difference between the two groups for repeat or auxiliary treatment $(\mathrm{OR}=0.34,95 \% \mathrm{CI}(0.03$ 3.96), $p=0.39$ ) (Figure 2(b)).

3.4. Hemoglobin Decreases. Four studies reporting operative hemoglobin decreases and comparing STPCNL to MTPCNL were included in this meta-analysis, and a significantly lower operative hemoglobin decrease was observed for STPCNL than MTPCNL (MD $=-0.46,95 \%$ CI $(-0.68$ to -0.25$)$, $p<0.0001$ ) (Figure 3(a)).
3.5. Blood Transfusion. Very few blood transfusion events were reported in five studies comparing single- versus multiple-tract PCNL. A meta-analysis by the fixed effects model $\left(I^{2}=0 \%\right)$ demonstrated a statistical difference between single- and multiple-tract PCNL with respect to blood transfusion $(\mathrm{OR}=0.48,95 \% \mathrm{CI}(0.34$ to 0.67$)$, $p<0.0001$ ) (Figure 3(b)).

3.6. Operative Time. Meta-analysis by the random effects model $\left(I^{2}=87 \%\right)$ demonstrated no significant difference between STPCNL and MTPCNL with respect to the operative time $(\mathrm{MD}=-42.78 \mathrm{~min}, 95 \% \mathrm{CI}(0-85.49$ to -0.07$)$, $p=0.05$ ) (Figure 3(c)).

3.7. Hospitalization Time. Regarding the length of inpatient stay, three studies were included in this meta-analysis. When 


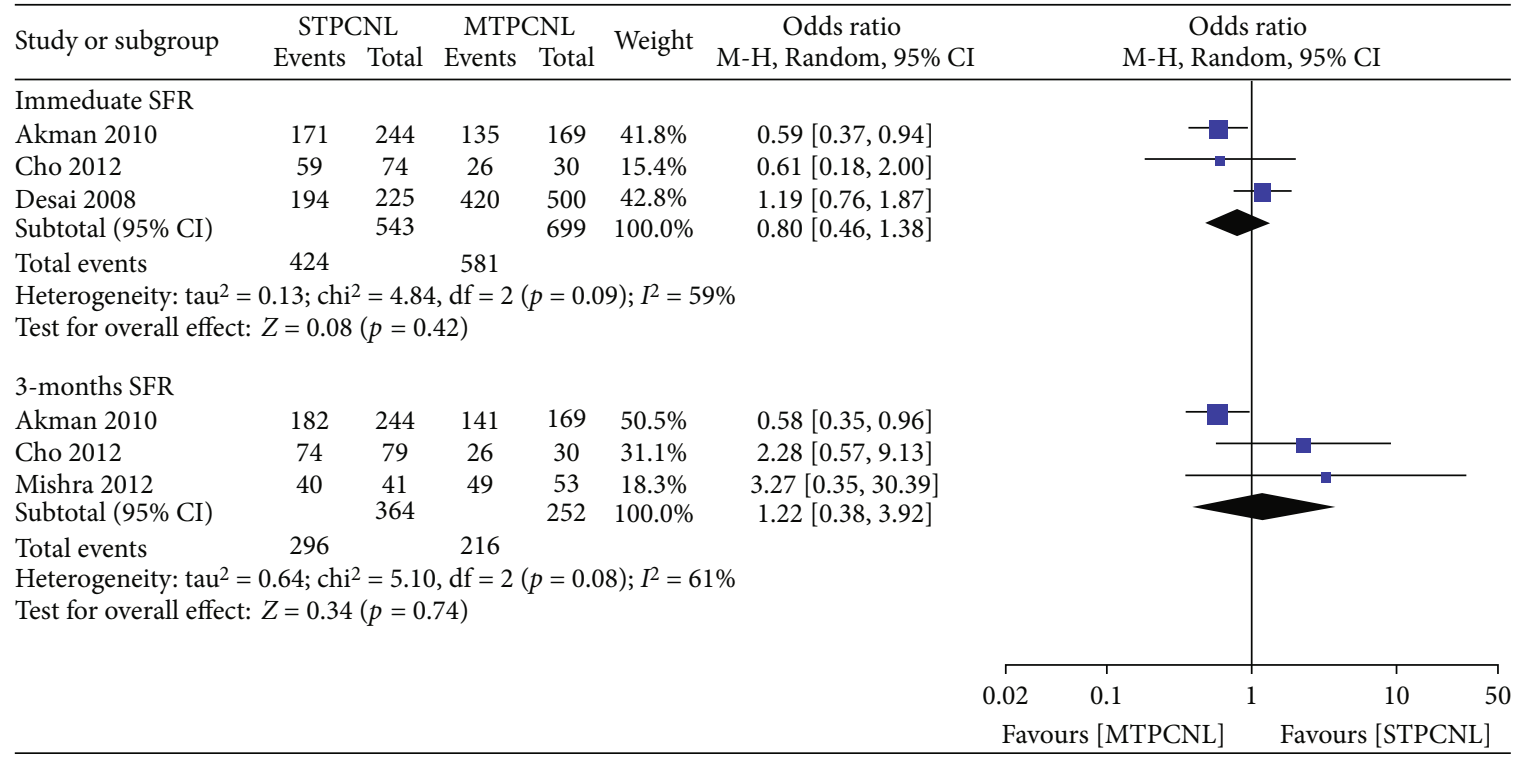

(a)

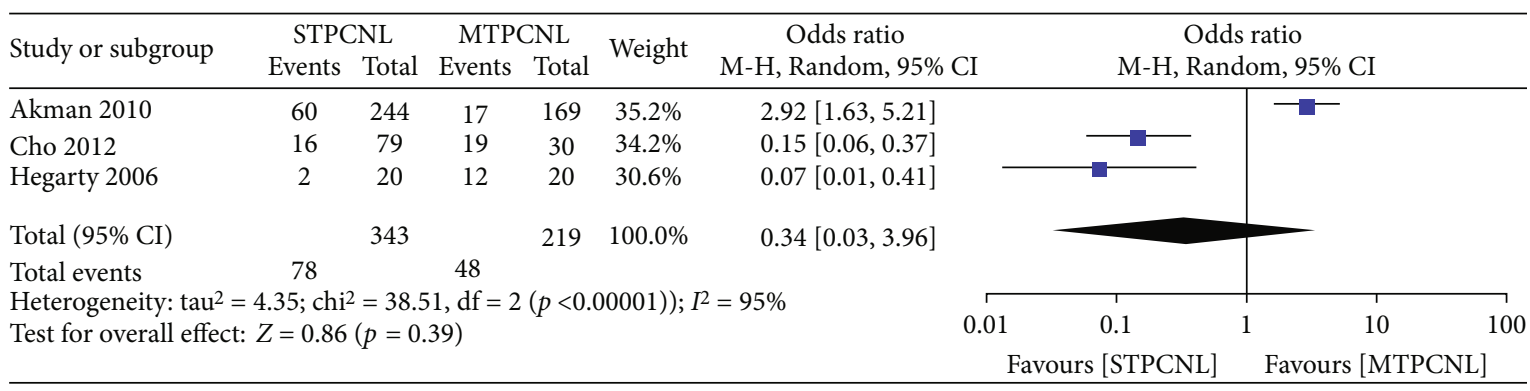

(b)

FIGURE 2: Forest plots comparing (a) immediate SFR and 3-month SFR and (b) auxiliary treatment.

pooled, the overall result showed that the STPCNL group and the MTPCNL group were similar with regard to this outcome $(\mathrm{MD}=-0.59,95 \% \mathrm{CI}(-3.59$ to 2.41$), p=0.70)$ (Figure $3(\mathrm{~d}))$.

3.8. Renal Function. Regarding serum creatinine, three studies comparing STPCNL to MTPCNL were included. On the basis of our analysis, no heterogeneity was found among the trials $\left(I^{2}=0\right)$; thus, a fixed effects model was selected. The meta-analysis showed no difference between STPCNL and MTPCNL (MD $=-0.02,95 \%$ CI (-0.06 to 0.02), $p=0.32$ ) (Figure 4).

3.9. Complications. Overall complications were reported in 4 studies. A total of 128 events were reported among 1116 participants. On the basis of our analysis, no heterogeneity was found among the trials $\left(I^{2}=0\right)$. The pooled analysis revealed no significant difference in the incidence of postoperative fever between the two groups $(\mathrm{OR}=0.74,95 \%$ CI $(0.49$ to 1.12 ), $p=0.15$ ) (Figure 5(a)). However, the combined overall result showed that STPCNL resulted in a lower risk of pulmonary complications $(\mathrm{OR}=0.28,95 \% \mathrm{CI}(0.09-0.83), p=$ 0.02) (Figure 5(b)). The meta-analyses detected no significant differences in other complications, such as fever $(\mathrm{OR}=0.86,95 \% \mathrm{CI}(0.27$ to 2.78$)$ (Figure $5(\mathrm{c})), p=0.80)$, urine leakage $(\mathrm{OR}=0.6,95 \% \mathrm{CI}(0.19$ to 1.87$), p=0.38)$
(Figure 5(d)), urinary tract infection $(\mathrm{OR}=0.85,95 \% \mathrm{CI}$ ( 0.13 to 5.45$), p=0.87$ ) (Figure $5(\mathrm{e})$ ), and nephrostomy time $(\mathrm{MD}=0.94,95 \% \mathrm{CI}(-2.39$ to 4.26$), p=0.58)$ (Figure $5(\mathrm{f}))$.

3.10. Publication Bias. A funnel plot was generated to assess publication bias (Figure 6). The result showed no apparent asymmetry, which indicated no obvious publication bias.

\section{Discussion}

Kidney calculi are a common urological disorder characterized by a high recurrence rate. Staghorn stones or complex caliceal calculi still represent an intractable challenge for urologists. PCNL is an integral component of the management of most staghorn and large-volume renal calculi [19]. The recently updated guidelines by the American Urological Association panel on staghorn calculi recommend the use of percutaneous monotherapy using multiple tracts as the preferred treatment option for most staghorn calculi. Although the safety of creating percutaneous renal tracts is well established, a concern regarding the use of multiple tracts remains due to additional complications compared to STPCNL. Although many studies have shown the effectiveness of single- versus multiple-tract PCNL for the treatment of complex caliceal calculi or staghorn stones, the results have been 


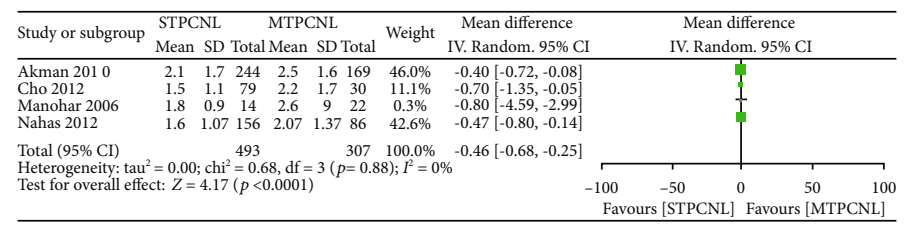

(a)

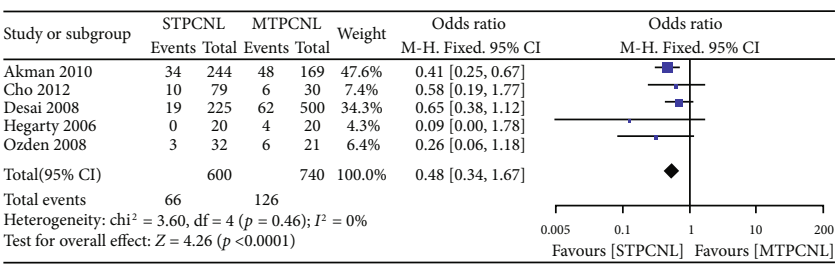

(b)

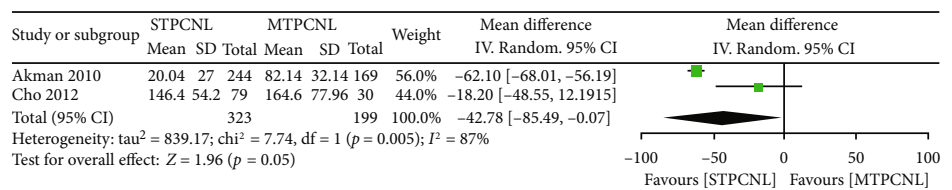

(c)

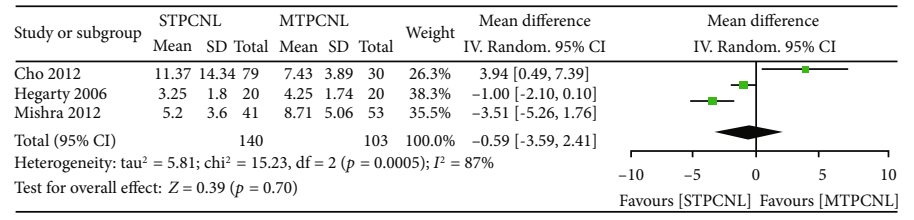

(d)

Figure 3: Forest plots comparing (a) hemoglobin decreases, (b) blood transfusion, (c) operation time, and (d) hospitalization time.

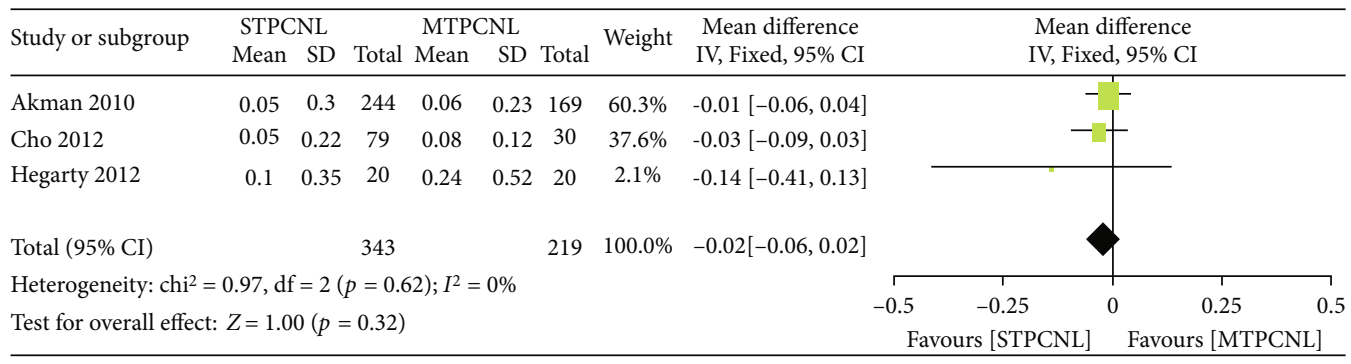

FIGURE 4: Forest plots of renal function.

controversial. The purpose of this meta-analysis was to evaluate and compare the efficacy and safety of single- versus multiple-tract PCNL in the surgical management of complex caliceal calculi or staghorn stones.

The SFR is the most important parameter for estimating the clinical efficacy of surgical methods for stones. In this meta-analysis, the pooled analysis revealed no significant difference in the SFR between the STPCNL and MTPCNL groups, indicating that STPCNL is an effective method for treating staghorn or complex caliceal calculi. However, many studies have shown the differential effectiveness of STPCNL and MTPCNL for the treatment of staghorn or complex caliceal calculi. After consulting relative literatures, the different results for the SFRs may be associated with the following factors. First, endourological societies have not agreed on a clear definition of the SFR, which has been defined as the presence of residual stones ranging from 0 to $4 \mathrm{~mm}$ in size. Second, SFRs may have been different if different tools were used for postoperative assessments. Some studies preferred to assess SFRs by KUB or ultrasound to identify clinically significant residual fragments while reducing radiation exposure to the patient [9]. However, evaluating the SFR using CT is more accurate as CT can better detect smaller residual stones [20]. In most of the literature, PCNL was primarily used as a part of combination therapy when managing staghorn or complex calculi [10]. Moreover, some studies have reported that the SFRs after PCNL monotherapy for staghorn stones ranged from $49 \%$ to $78 \%$ [21, 22]. Streem et al. [23] reported an SFR of $63-70 \%$ when they used "sandwich therapy" with extracorporeal shockwave lithotripsy (ESWL), where PCNL 


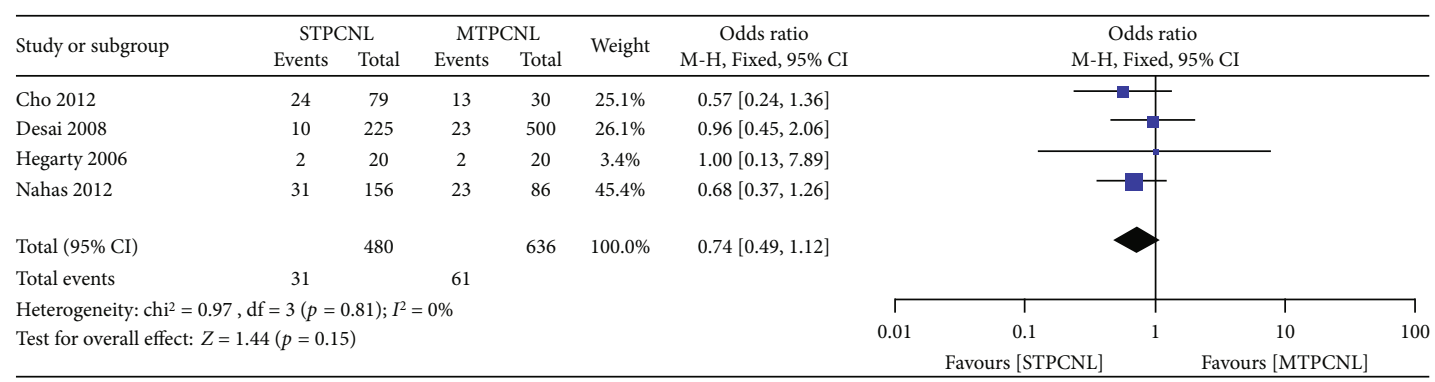

(a)

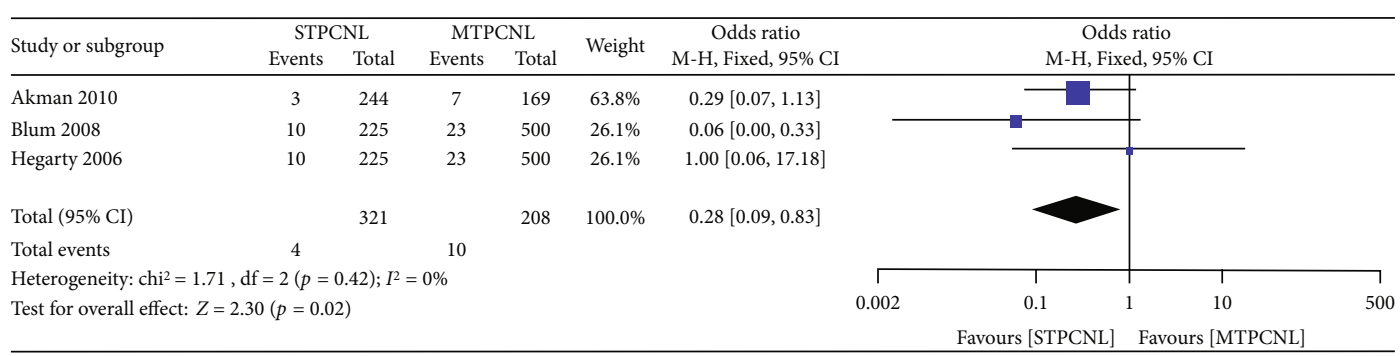

(b)

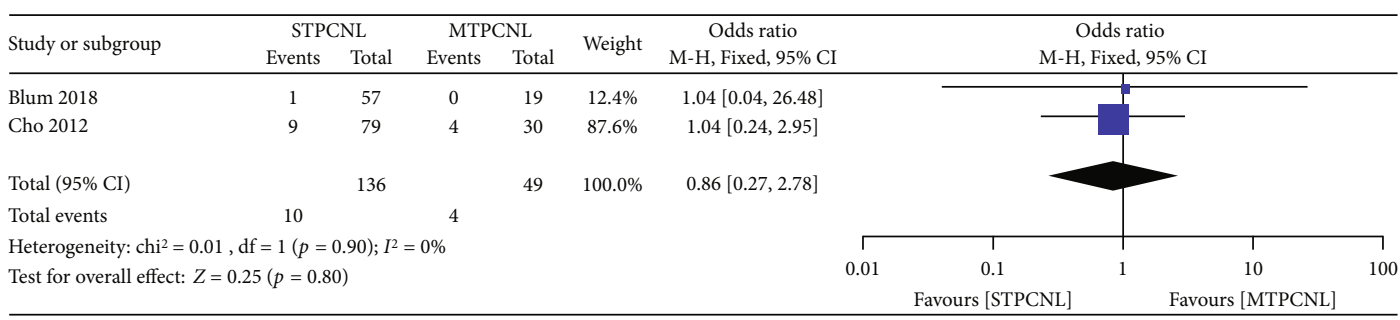

(c)

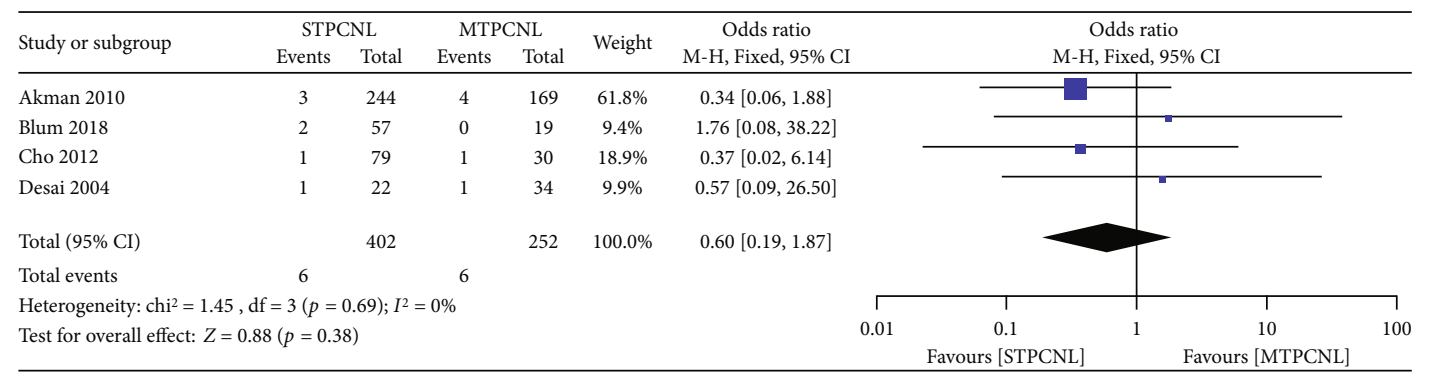

(d)

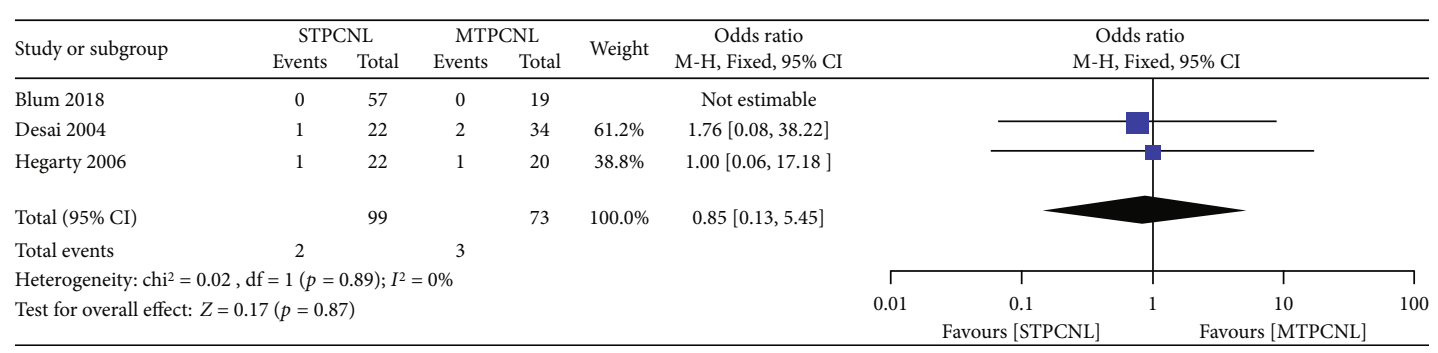

(e)

FIgURe 5: Continued. 


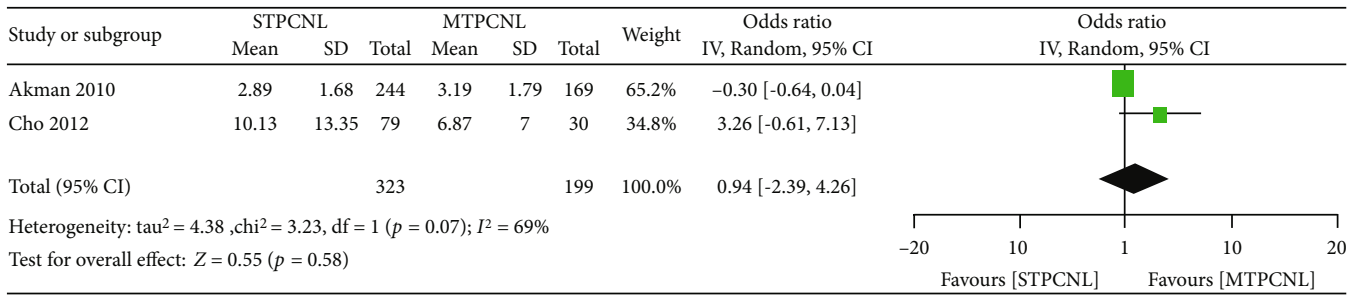

(f)

FIGURE 5: Forest plots of other parameters including (a) complications, (b) pulmonary complications, (c) postoperative fever, (d) urine leakage, (e) urinary tract infection, (f) nephrostomy time.

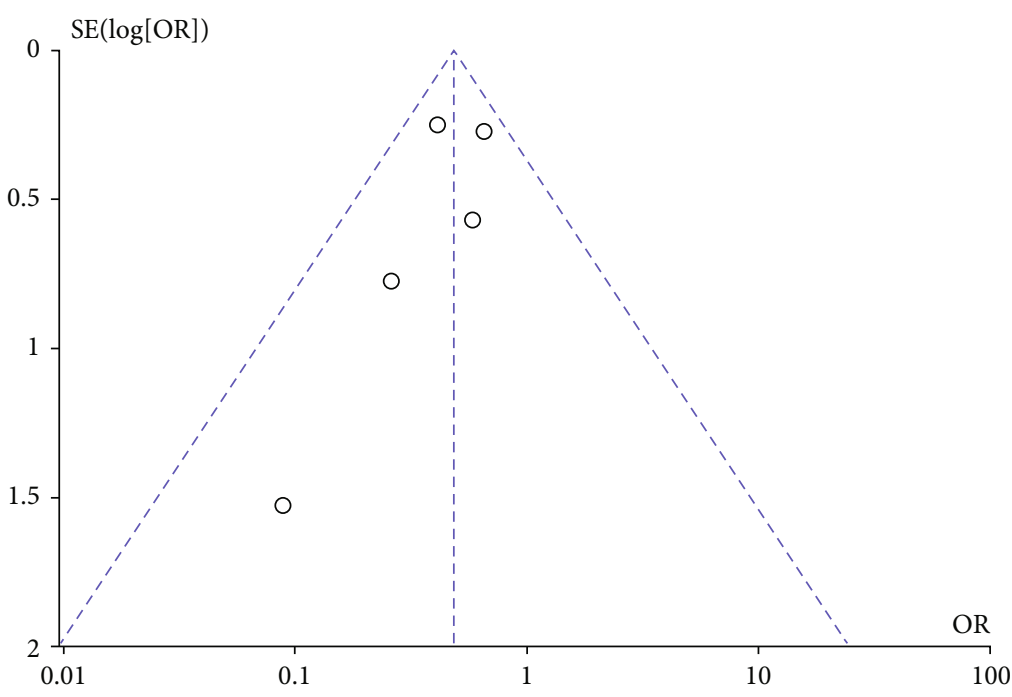

Figure 6: Funnel plot for evaluation of potential publication bias.

was the terminal procedure (PCNL-ESWL-PCNL). In addition, several sessions of PCNL may be necessary to remove all stone branches [24], and repeat or auxiliary treatment such as ESWL might be required for residual fragments to ensure the SFR. The SFR is also related to the follow-up time as the final SFR after surgery is higher than that immediate SFR after surgery. Time is needed for stone fragments to be flushed out with urine. Some studies involved treatment for complete staghorn stones, while other studies included partial and complete staghorn stones. Unfortunately, we were unable to conduct a subgroup analysis to analyze the influence of the number of tracts on treatment effectiveness due to insufficient data. Last, certain points of surgical techniques merit special emphasis regarding the SFR. Renal access should be established by endourologists with considerable experience in percutaneous surgery because they will be most familiar with the pelvicalyceal anatomy and the surgical procedure. Accordingly, more studies are needed to obtain more reliable outcomes.

Major complications of PCNL include bleeding. The results of this meta-analysis showed that the use of multiple tracts contributed to a higher hemoglobin drop and a higher frequency of transfusions. These results are consistent with previously published results. Hemorrhage is generally associated with the initial puncture and injury of renal blood vessels and the surrounding organs. Additional tracts during
PCNL may increase the risk of injury to major blood vessels and may complicate recovery from puncture injury. In this meta-analysis, we analyzed only the overall blood loss and transfusion events, but we did not analyze subgroups for the number of tracts due to the low amount of data from the included studies due to insufficient data. However, in some studies, multiple tracts did not significantly increase blood loss and transfusion requirements. Hegarty and Desai noted a mean drop in hemoglobin in patients with multiple tracts similar to that in patients with single tracts $[12,14$, 25]. They thought that this result was probably related to lower baseline hemoglobin concentrations, and transfusions were performed on the second or third postoperative day rather than as an emergency for significant blood loss. Although an increase in the number of tracts has adverse effects in terms of blood loss, many measures can be implemented to avoid more blood loss. First, direct puncture into the pelvis or near the infundibular neck may be avoided to reduce the risk of severe bleeding [26]. Second, selecting the size of the tract based on the width of the funnel and the angle at which the tract enters the renal pelvis may help prevent the overdilatation of the infundibulum and subsequent significant bleeding [15]. In addition, staging the procedure may strengthen the urethra, rendering PCNL surgery relatively simple without massive blood loss [5]. Furthermore, based on our experience, we suggest using gentle techniques, 
avoiding manipulations such as levering the nephroscope and using flexible endoscopes and nitinol baskets when necessary to prevent bleeding complications. Finally, some doctors have suggested that the use of balloon dilators was associated with lower blood loss. The use of Amplatz dilators may be a reason for relatively high blood loss.

For complex or multiple stones, more than one percutaneous access may be required for stone disintegration, potentially increasing the risk of parenchymal injury and reducing kidney function [27]. Although we found no significant difference in renal function based on serum creatinine, a trend toward MTPCNL being associated with a greater reduction in renal function was noted. Some authors have found that multiaccess PCNL was associated with a significant decrease in the renal function of the targeted kidney according to MAG3 nuclear renogram results compared to a single-tract approach [13]. Therefore, utilizing a minimal number or smaller PCNL tracts that achieve optimal stone removal with the least degree of injury to the renal parenchyma is advisable. Although patients undergoing MTPCNL may demonstrate a postoperative decline in renal function, some studies have noted that this change was temporary and reversible. The authors indicated a statistically significant improvement based on the glomerular filtration rate (eGFR) at the first postoperative year, which worsened in only $6.8 \%$ of patients with a solitary kidney who had been treated with PCNL [8]. Some authors thought that increases in creatinine levels at an early postoperative period were temporary and probably associated with anesthetic agents and medications $[14,25]$. Patients with stone disease and baseline renal insufficiency ultimately show improvements in renal function as a result of the relief of obstruction and resolution of infection with complete calculus clearance $[5,28]$. Numerous studies have found no deleterious effects of PCNL on renal function among many patients, including multiple-access procedures $[11,29,30]$. However, renal function is generally assessed with different methods, such as the eGFR or serum creatinine, the efficacy of which can be substantially influenced by patient factors, including body mass index (BMI), baseline renal function, and ethnicity [31-33]. Furthermore, the eGFR may serve as an estimate of global nephron function but not for unilateral kidney PCNL; only a single kidney unit is affected. Recently, nuclear renography using the radioisotope technetium-99m mercaptoacetyltriglycine (99mTc-MAG3) has been commonly used to assess urinary obstruction as well as the relative percent function of each kidney. One of the limitations of this meta-analysis was the small number of studies with detailed data for more than two tracts. In fact, most multiple-tract PCNLs had only two access tracts. Thus, we cannot conduct a subgroup analysis to analyze the number of access tracts and the influence of the number of tracts on renal function.

When comparing morbidity between single and multiple tracts, the blood transfusion rate was higher in patients with multiple tracts, but most complications were similar and not significantly different between groups in our meta-analysis. However, we found that multiple-tract PCNL may lead to higher rates of pulmonary complications. Upper-pole (UP) access is well documented to be associated with a higher risk of thoracic complications such as pneumothorax and hydrothorax, especially with punctures that originate above the 11th rib [34-36]. Pulmonary injury due to lung transgression can occur even with controlled expirations anywhere from $14 \%$ on the left side to $29 \%$ on the right side $[37,38]$. The rate of pulmonary complications is unequivocally higher with supracostal UP approaches, with some sources describing occurrence rates greater than 15\% [39]. Despite this finding, the literature tends to support the continued use of UP access citing more expeditious, direct, and complete stone removal with fewer access sites [9]. After reviewing many studies, many measures can reduce the rates of complications. First, for supracostal UP access, the puncture site can be localized in the midpoint of the 11th and 12th intercostal spaces, usually 1 to $2 \mathrm{~cm}$ cranial to the upper pole of the most medial calix [8]. The advantages of direct UP access are good exposure to most of the calices and the renal pelvis, the possibility of reaching the ureteropelvic junction and upper ureter, and the ability to operate along the long axis of the kidney, which causes less torque of the rigid nephroscope and ultimately less bleeding. In our experience, access through a lower calix into the upper and middle calix is difficult and necessitates longer operative and fluoroscopy times. Second, when deciding to use MTPCNL, placing all the tracts and fixing all guidewires before starting dilatation of the first tract are advisable [17]. In addition, preoperative planning of the procedure and selecting the appropriate technique must be individualized for each patient. On the basis of our assessment of calculus configuration and collecting system anatomy, all possible percutaneous tracts were punctured right at the outset, and guidewires were secured because confirming correct percutaneous needle placement is significantly easier in an intact collecting system [10].

To the best of our knowledge, we are the first to evaluate the current evidence on the effectiveness and safety of singleversus multiple-tract PCNL in the surgical management of complex caliceal calculi and staghorn stones following the PRISMA guidelines. However, limitations should be considered when drawing conclusions. First, because most of the included studies were retrospective, biases from the original studies, such as selection bias, information bias, and other confounding factors, likely have not been excluded in this meta-analysis. Although all ten eligible studies involving 1844 patients were of moderate quality (scores $\geq 7$ ) according to the NOS, bias still exists, which might render these results less reliable.

Second, in addition to the surgical process, other important clinical parameters, such as stone size, complete or incomplete staghorn stones, stone location, and stone composition, were important factors influencing effectiveness and safety. Therefore, conducting subgroup analyses to compare the efficacy of these two approaches may have rendered the findings more generalizable. Unfortunately, we were unable to conduct a subgroup analysis to analyze the influence of the number of tracts on effectiveness due to insufficient data. Therefore, we hope that intercalating additional data will shed light on these outcomes in the future.

Finally, despite the well-recognized advantages of metaanalyses, the results were predictably affected by the quality 
of the included studies and reporting biases, which may have occurred due to the lack of studies confirming the null hypothesis or publishing nonsignificant outcomes. Studies reporting nonsignificant outcomes have historically been more difficult to publish than studies showing statistically significant results. One would hope that as the publication process matures, this evidence base will become more sophisticated, which may limit the influence of publication bias. Given these limitations, we hope that well-designed prospective trials are designed to verify the findings of this meta-analysis in the future.

Despite these limitations, this study represents the first meta-analysis comparing STPCNL and MTPCNL in the surgical management of staghorn or complex caliceal calculi. Thus, we provide the most up-to-date information on the surgical treatment of patients with complex caliceal calculi or staghorn stones, which we hope can provide some help to urologists and patients when selecting the optimal therapy. However, the findings of this study should be further confirmed by well-designed prospective randomized controlled trials (RCTs) with a larger patient series.

\section{Conclusion}

Our systematic review and meta-analysis demonstrated that STPCNL seems to be a safe and feasible alternative compared to MTPCNL for patients with staghorn stones and has many advantages, such as no decreases in the final SFR, fewer blood transfusions, and even fewer complications, such as pulmonary complications and postoperative fever. However, our conclusion should be treated prudently, and further large-sample, prospective, and multicenter studies and RCTs should be undertaken to confirm our findings.

\section{Abbreviations}

AMSTAR: Assessing the methodological quality of systematic reviews

CI: $\quad$ Confidence interval

ESWL: Extracorporeal shockwave lithotripsy

LE: $\quad$ Level of evidence

MD: $\quad$ Mean difference

MTPCNL: Multiple tract percutaneous nephrolithotomy

OR: Odds ratio

PCNL: Standard percutaneous nephrolithotomy

PRISMA: Preferred Reporting Items for Systematic Reviews and Meta-Analyses

RCT: $\quad$ Randomized control trial

STPCNL: Single-tract percutaneous nephrolithotomy

SFR: $\quad$ Stone-free rate

UP: $\quad$ Upper pole

URL: Transurethral ureteroscope lithotripsy.

\section{Data Availability}

The datasets used and/or analyzed during the current study are available from the corresponding author on reasonable request.

\section{Conflicts of Interest}

The authors declare that no conflicts of interest exist.

\section{Authors' Contributions}

Binbin Jiao, Zhenshan Ding, and Zhenkai Luo contributed as the co-first author.

\section{References}

[1] A. P. Ganpule and M. Desai, "Management of the staghorn calculus: multiple-tract versus single-tract percutaneous nephrolithotomy," Current Opinion in Urology, vol. 18, no. 2, pp. 220-223, 2008.

[2] G. M. Preminger, D. G. Assimos, J. E. Lingeman et al., "Chapter 1: AUA guideline on management of staghorn calculi: diagnosis and treatment recommendations," The Journal of Urology, vol. 173, no. 6, pp. 1991-2000, 2005.

[3] R. K. Handa, A. P. Evan, L. R. Willis et al., "Renal functional effects of multiple-tract percutaneous access," Journal of Endourology, vol. 23, no. 12, pp. 1951-1956, 2009.

[4] M. Aron, R. Yadav, R. Goel et al., "Multi-tract percutaneous nephrolithotomy for large complete staghorn calculi," Urologia Internationalis, vol. 75, no. 4, pp. 327-332, 2005.

[5] R. Kukreja, M. Desai, S. Patel, S. Bapat, and M. Desai, "Factors affecting blood loss during percutaneous nephrolithotomy: prospective study," Journal of Endourology, vol. 18, no. 8, pp. 715-722, 2004.

[6] B. Phillips, C. Ball, D. Sackett, D. Badenoch, S. Straus, and B. Haynes, "Oxford centre for evidence-based medicineLevels of Evidence," 2009, February 2020, https://www.cebm .net/2009/06/oxford-centre-evidence-based-medicine-levelsevidence-march-2009.

[7] G. A. Wells, B. Shea, D. O'Connell et al., The Newcastle Ottawa Scale (NOS) for assessing the quality of nonrandomized studies in meta analyses, Ottawa Hospital Research Institute, 2016, May 2016, http://www.ohri.ca/programs/clinical_ epidemiology/oxford.asp.

[8] T. Akman, E. Sari, M. Binbay et al., "Comparison of outcomes after percutaneous nephrolithotomy of staghorn calculi in those with single and multiple accesses," Journal of Endourology, vol. 24, no. 6, pp. 955-960, 2010.

[9] K. A. Blum, E. Parkhomenko, J. Thai, T. Tran, and M. Gupta, "A contemporary lower pole approach for complete staghorn calculi: outcomes and efficacy," World Journal of Urology, vol. 36, no. 9, pp. 1461-1467, 2018.

[10] M. R. Desai, R. A. Kukreja, S. H. Patel, and S. D. Bapat, "Percutaneous nephrolithotomy for complex pediatric renal calculus disease," Journal of Endourology, vol. 18, no. 1, pp. 23-27, 2004.

[11] H. J. Cho, J. Y. Lee, S. W. Kim, T. K. Hwang, and S. H. Hong, "Percutaneous nephrolithotomy for complex renal calculi: is multi-tract approach ok?," The Canadian Journal of Urology, vol. 19, no. 4, pp. 6360-6365, 2012.

[12] M. Desai, A. Ganpule, and T. Manohar, "'Multiperc" for complete staghorn calculus," Journal of Endourology, vol. 22, no. 9, pp. 1831-1834, 2008.

[13] E. Ozden, A. Sahin, B. Tan, H. S. Doğan, M. T. Eren, and S. Tekgül, "Percutaneous renal surgery in children with complex stones," Journal of Pediatric Urology, vol. 4, no. 4, pp. $295-298,2008$. 
[14] N. J. Hegarty and M. M. Desai, "Percutaneous nephrolithotomy requiring multiple tracts: comparison of morbidity with single-tract procedures," Journal of Endourology, vol. 20, no. 10 , pp. $753-760,2006$.

[15] T. Manohar, A. P. Ganpule, P. Shrivastav, and M. Desai, "Percutaneous nephrolithotomy for complex caliceal calculi and staghorn stones in children less than 5 years of age," Journal of Endourology, vol. 20, no. 8, pp. 547-551, 2006.

[16] S. Mishra, R. B. Sabnis, and M. Desai, "Staghorn morphometry: a new tool for clinical classification and prediction model for percutaneous nephrolithotomy monotherapy," Journal of Endourology, vol. 26, no. 1, pp. 6-14, 2012.

[17] A. R. El-Nahas, I. Eraky, A. A. Shokeir et al., "Percutaneous nephrolithotomy for treating staghorn stones: 10 years of experience of a tertiary-care centre," Arab Journal of Urology, vol. 10, no. 3, pp. 324-329, 2012.

[18] X. Wan, W. Wang, J. Liu, and T. Tong, "Estimating the sample mean and standard deviation from the sample size, median, range and/or interquartile range," BMC Medical Research Methodology, vol. 14, no. 1, 2014.

[19] M. Singla, A. Srivastava, R. Kapoor et al., "Aggressive approach to staghorn calculi-safety and efficacy of multiple tracts percutaneous nephrolithotomy," Urology, vol. 71, no. 6, pp. 10391042, 2008.

[20] Y. Osman, N. el-Tabey, H. Refai et al., "Detection of residual stones after percutaneous nephrolithotomy: role of nonenhanced spiral computerized tomography," The Journal of Urology, vol. 179, no. 1, pp. 198-200, 2008.

[21] K. M. Al-Kohlany, A. A. Shokeir, A. Mosbah et al., "Treatment of complete staghorn stones: a prospective randomized comparison of open surgery versus percutaneous nephrolithotomy," The Journal of Urology, vol. 173, no. 2, pp. 469473, 2005.

[22] F. Soucy, R. Ko, M. Duvdevani, L. Nott, J. D. Denstedt, and H. Razvi, "Percutaneous nephrolithotomy for staghorn calculi: a single center's experience over 15 years," Journal of Endourology, vol. 23, no. 10, pp. 1669-1673, 2009.

[23] S. B. Streem, A. Yost, and B. Dolmatch, "Combination "sandwich" therapy for extensive renal calculi in 100 consecutive patients: immediate, long-term and stratified results from a 10-year experience," The Journal of Urology, vol. 158, no. 2, pp. 342-345, 1997.

[24] M. Desai, P. Jain, A. Ganpule, R. Sabnis, S. Patel, and P. Shrivastav, "Developments in technique and technology: the effect on the results of percutaneous nephrolithotomy for staghorn calculi," BJU International, vol. 104, no. 4, pp. 542548, 2009.

[25] A. P. Ganpule, S. Mishra, and M. R. Desai, "Multiperc versus single perc with flexible instrumentation for staghorn calculi," Journal of Endourology, vol. 23, no. 10, pp. 1675-1678, 2009.

[26] Z. Guohua, W. Zhong, X. Li et al., "Minimally invasive percutaneous nephrolithotomy for staghorn calculi: a novel single session approach via multiple 14-18Fr tracts," Surgical Laparoscopy, Endoscopy \& Percutaneous Techniques, vol. 17, no. 2, pp. 124-128, 2007.

[27] Y. Zhou, A. Gurioli, J. Luo et al., "Comparison of effect of minimally invasive percutaneous nephrolithotomy on split renal function: single tfractvsmultiple tracts," Journal of Endourology, vol. 31, no. 4, pp. 361-365, 2017.

[28] M. Gupta, D. M. Bolton, P. N. Gupta, and M. L. Stoller, "Improved renal function following aggressive treatment of urolithiasis and concurrent mild to moderate renal insufficiency," The Journal of Urology, vol. 152, no. 4, pp. 10861090, 1994.

[29] N. A. el-Tabey, A. R. el-Nahas, I. Eraky et al., "Long-term functional outcome of percutaneous nephrolithotomy in solitary kidney," Urology, vol. 83, no. 5, pp. 1011-1015, 2014.

[30] K. Wood, T. Keys, P. Mufarrij, and D. G. Assimos, "Impact of stone removal on renal function: a review," Revista de Urología, vol. 13, no. 2, pp. 73-89, 2011.

[31] M. Froissart, J. Rossert, C. Jacquot, M. Paillard, and P. Houillier, "Predictive performance of the modification of diet in renal disease and Cockcroft-Gault equations for estimating renal function," Journal of the American Society of Nephrology, vol. 16, no. 3, pp. 763-773, 2005.

[32] E. D. Poggio, X. Wang, T. Greene, F. van Lente, and P. M. Hall, "Performance of the modification of diet in renal disease and Cockcroft-Gault equations in the estimation of GFR in health and in chronic kidney disease," Journal of the American Society of Nephrology, vol. 16, no. 2, pp. 459-466, 2005.

[33] S. Mahajan, G. K. Mukhiya, R. Singh et al., "Assessing glomerular filtration rate in healthy Indian adults: a comparison of various prediction equations," Journal of Nephrology, vol. 18, no. 3, pp. 257-261, 2005.

[34] H. N. Shah, S. S. Hegde, J. N. Shah, and M. B. Bansal, "Safety and efficacy of supracostal access in tubeless percutaneous nephrolithotomy," Journal of Endourology, vol. 20, no. 12, pp. 1016-1021, 2006.

[35] S. Sukumar, B. Nair, P. G. Kumar, K. V. Sanjeevan, and H. S. Bhat, "Supracostal access for percutaneous nephrolithotomy: less morbid, more effective," International Urology and Nephrology, vol. 40, no. 2, pp. 263-267, 2008.

[36] D. Olvera-Posada, T. Tailly, H. Alenezi et al., "Risk factors for postoperative complications of percutaneous nephrolithotomy at a tertiary referral center," The Journal of Urology, vol. 194, no. 6, pp. 1646-1651, 2015.

[37] R. Munver, F. C. Delvecchio, G. E. Newman, and G. M. Preminger, "Critical analysis of supracostal access for percutaneous renal surgery," The Journal of Urology, vol. 166, no. 4, pp. 1242-1246, 2001.

[38] K. D. Hopper and W. F. Yakes, "The posterior intercostal approach for percutaneous renal procedures: risk of puncturing the lung, spleen, and liver as determined by CT," AJR. American Journal of Roentgenology, vol. 154, no. 1, pp. 115117, 1990.

[39] B. Lojanapiwat and S. Prasopsuk, "Upper-pole access for percutaneous nephrolithotomy: comparison of supracostal and infracostal approaches," Journal of Endourology, vol. 20, no. 7, pp. 491-494, 2006. 